Pacific Journal of Mathematic 


\title{
TOPOLOGIES ON THE TORSION-THEORETIC SPECTRUM OF A NONCOMMUTATIVE RING
}

\author{
JONATHAN S. GOLAN
}

\begin{abstract}
Let $R$-sp be the collection of all prime torsion theories on the category of left $R$-modules over an associative ring $R$. Three topologies - the order topology, the finitary order topology, and the reverse order topology (in the case that $R$ is left noetherian) - are defined on $R$-sp and each is shown to exhibit some properties of the Zariski topology on the spectrum of a commutative ring. A fourth topology - the Gillman topology - is defined on $R$-sp when $R$ is left noetherian and is used to characterize the separation of the reverse order topology.
\end{abstract}

1. Background and notation. Throughout the following $R$ will always denote an associative ring with unit element 1 . Unless the contrary is specifically stated, all modules and morphisms will be taken from the category $R$-mod of unitary left $R$-modules. Homomorphisms will be written as acting on the side opposite scalar multiplication, i.e., on the right. The injective hull of a module $M$ will be denoted by $E(M)$.

The term "torsion theory" will always be used to mean hereditary torsion theory in the sense of [2]. In this section we summarize the information about torsion theories which we will need. The reader is referred to $[2,4,6,10]$ for further elucidation and for proofs.

A torsion theory $\tau$ can be completely characterized by any of the following data, each of which uniquely determines all of the others:

(i) The class $\mathscr{T}_{\tau}$ of torsion modules. This class is closed under taking submodules, factor modules, direct sums, and extensions (i.e., if $0 \rightarrow M^{\prime} \rightarrow M \rightarrow M^{\prime \prime} \rightarrow 0$ is an exact sequence with $M^{\prime}, M^{\prime \prime} \in \mathscr{T}_{\tau}$, then $\left.M \in \mathscr{T}_{\tau}\right)$.

(ii) The class $\mathscr{F}_{\tau}$ of torsion-free modules. This class is closed under taking submodules, injective hulls, direct products, and extensions.

(iii) The set $\mathscr{L}_{\tau}$ of left ideals $I$ of $R$ satisfying $R / I \in \mathscr{T}_{\tau}$. This set is an idempotent filter, i.e., if $I \in \mathscr{L}_{\tau}$ then so does every left ideal of $R$ properly containing $I$ and so does $(I: r)=\left\{r^{\prime} \in R \mid r^{\prime} r \in I\right\}$ for every $r \in R$. Furthermore, $\mathscr{L}_{\tau}$ is closed under taking finite intersections and, if $I \in \mathscr{L}_{\tau}$ and $(H: r) \in \mathscr{L}_{\tau}$ for every $r \in I$ then $H \in \mathscr{L}_{\tau}$.

(iv) The class $\mathscr{E}_{\tau}$ of absolutely pure modules. These are elements $N$ of $\mathscr{F}_{\tau}$ satisfying the condition that if $N$ is a submodule of $M \in \mathscr{F}_{\tau}$ then $M / N \in \mathscr{F}_{\tau}$. The full subcategory of $R$-mod defined by $\mathscr{E}_{\tau}$ is abelian. 
(v) The functor $T_{\tau}(-): R$-mod $\rightarrow R$-mod which assigns to each module $M$ the (unique) submodule $T_{\tau}(M)$ of $M$ satisfying $T_{\tau}(M) \in \mathscr{T}_{\tau}$ and $M / T_{\tau}(M) \in \mathscr{F}_{\tau}$.

(vi) The functor $Q_{\tau}(\ldots): R$-mod $\rightarrow \mathscr{E}_{\tau}$ which is the left adjoint of the inclusion functor.

For any module $M$, the module $Q_{\tau}(M)$ is called the localization of $M$ with respect to the torsion theory $\tau$. The endomorphism ring of $Q_{\tau}(R)$ is called the quotient ring of $R$ with respect to $\tau$ and will be denoted by $R_{\tau}$. As left $R$-modules, $Q_{\tau}(R)$ and $R_{\tau}$ are isomorphic. Furthermore, every module $Q_{\tau}(M)$ is canonically a left $R_{\tau}$-module. For each left $R$-module $M$ we have a canonical $R$-homomorphism $\hat{\tau}_{M}: M \rightarrow Q_{\tau}(M)$.

The class of all hereditary torsion theories on $R$-mod will be denoted by $R$-tors. This class can be partially ordered by setting $\tau \leqq \tau^{\prime}$ if and only if $\mathscr{T}_{\tau} \subseteq \mathscr{T}_{\tau^{\prime}}$. If $\left\{\tau_{i} \mid i \in \Omega\right\}$ is a family of torsion theories then we denote the largest torsion theory less than or equal to all of them by $\Lambda_{i \in \Omega} \tau_{i}$. Such a theory always exists and is defined by

$$
\mathscr{T}_{\wedge \tau_{i}}=\bigcap \mathscr{T}_{\tau_{i}} .
$$

Similarly we denote by $\mathbf{V}_{i \in \Omega} \tau_{i}$ the smallest torsion theory greater than or equal to all of the $\tau_{i}$. This theory always exists and is defined by

$$
\mathscr{F}_{v_{\tau_{i}}}=\bigcap \mathscr{F}_{\tau_{i}} .
$$

The class $R$-tors has a minimal element $\xi$, defined by $\mathscr{T}_{\xi}=\{0\}$, and a maximal element $\chi$, defined by $\mathscr{F}_{\chi}=\{0\}$. A torsion theory $\tau$ which is not equal to $\chi$ is called proper; a torsion theory $\tau$ which is not equal to $\xi$ is called nontrivial. The collection of all proper torsion theories on $R$-mod will be denoted by $R$-prop.

If $\mathscr{A}$ is any family of modules then we denote by $\xi(\mathscr{A})$ the smallest torsion theory in which every $M \in \mathscr{A}$ is torsion and by $\chi(\mathscr{A})$ the largest torsion theory in which every $M \in \mathscr{A}$ is torsion free. Then $\mathscr{F}_{\xi(\mathscr{N})}=\left\{N \mid \operatorname{Hom}_{R}(M, E(N))=0\right.$ for all $\left.M \in \mathscr{A}\right\}$ and $\mathscr{T}_{\chi(\mathscr{A})}=$ $\left\{M \mid \operatorname{Hom}_{R}(M, E(N))=0\right.$ for all $\left.N \in \mathscr{A}\right\}$. Furthermore, for any $\tau \in$ $R$-tors, we have $\tau=\mathrm{V}\left\{\xi(R / I) \mid I \in \mathscr{L}_{\tau}\right\}$.

Lemma 1.1. Let $I, I^{\prime}$ be left ideals of $R$. Then

$$
\xi(R / I) \vee \xi\left(R / I^{\prime}\right)=\xi\left(R /\left[I \cap I^{\prime}\right]\right) \text {. }
$$

Proof. Let $\tau=\xi(R / I) \vee \xi\left(R / I^{\prime}\right)$. Then $N \in \mathscr{F}_{\tau}$ if and only if $\operatorname{Hom}_{R}(R / I, E(N))=\operatorname{Hom}_{R}\left(R / I^{\prime}, E(N)\right)=0$. Clearly this holds if $N \in \mathscr{F}_{\xi\left(R /\left[I \cap I^{\prime}\right]\right)}$. Conversely, assume that $N \in \mathscr{F}_{\tau}^{-}$and $\alpha \in$ $\operatorname{Hom}_{R}\left(R /\left[I \cap I^{\prime}\right], E(N)\right)$. Then we have a canonical monomorphism 
$\theta: R /\left[I \cap I^{\prime}\right] \rightarrow R / I \oplus R / I^{\prime}$. Since $E(N)$ is injective, there then exists a homomorphism $\beta: R / I \oplus R / I^{\prime} \rightarrow E(N)$ with $\alpha=\theta \beta$. Since $N \in \mathscr{F}_{\tau}$ we must have $\beta=0$ whence $\alpha=0$, proving $N \in \mathscr{F}_{\xi\left(R /\left[I \cap I^{\prime}\right]\right)}$.

For any module $M$, we define the wide support of $M$ by wsupp $(M)=$ $\left\{\tau \in R\right.$-prop $\left.\mid M \notin \mathscr{T}_{\tau}\right\}=\left\{\tau \in R\right.$-prop $\left.\mid Q_{\tau}(M) \neq 0\right\}$. The following lemma follows directly from this definition.

LeMma 1.2. For a module $M$,

(1) $\quad M=\Sigma M_{i}$ implies that $\operatorname{wsupp}(M)=\mathrm{U} \operatorname{wsupp}\left(M_{i}\right)$.

(2) $N \cong M$ implies that $w \operatorname{supp}(M)=w \operatorname{wupp}(N) \cup \operatorname{wsupp}(M / N)$.

2. The order topologies on $R$-prop. We define functions

$$
R \text {-tors } \stackrel{d}{\stackrel{d}{\longrightarrow}} \text { subsets of } R \text {-prop }
$$

as follows

$$
\begin{aligned}
& c: \tau \longmapsto\left\{\tau^{\prime} \in R \text {-prop } \mid \tau \leqq \tau^{\prime}\right\} \\
& d: U \longmapsto \wedge U .
\end{aligned}
$$

Then we clearly have $d c(\tau)=\tau$ for all $\tau \in R$-tors (making the convention that $\wedge \varnothing=\chi)$.

LemMa 2.1. If $\left\{\tau_{i} \mid i \in \Omega\right\} \cong R$-tors then

(1) $\tau_{i_{1}} \leqq \tau_{i_{2}}$ implies that $c\left(\tau_{i_{1}}\right) \supseteqq c\left(\tau_{i_{2}}\right)$.

(2) $c\left(\tau_{i_{1}} \wedge \tau_{i_{2}}\right) \supseteqq c\left(\tau_{i_{1}}\right) \cup c\left(\tau_{i_{2}}\right)$.

(3) $c\left(\vee \tau_{i}\right)=\bigcap c\left(\tau_{i}\right)$.

Proof. (1) follows directly from the definition. By (1), we have $c\left(\tau_{i_{j}}\right) \subseteq c\left(\tau_{i_{1}} \wedge \tau_{i_{2}}\right)$ for $j=1,2$ which implies (2). As for (3), if $\tau \epsilon$ $\bigcap c\left(\tau_{i}\right)$ then $\tau \geqq \tau_{i}$ for all $i \in \Omega$ and so, by definition, $\tau \geqq \bigvee \tau_{i}$, which is to say that $\tau \in c\left(\vee \tau_{i}\right)$. The reverse inclusion is trivial.

The proof of the following proposition is based on [1].

Proposition 2.2. If $R$ is left noetherian and if $\tau \in R$-prop then $c(\tau)$ contains a maximal element of $R$-prop.

Proof. Let $\mathscr{A}$ be the class of all proper ideals $I$ of $R$ satisfying the conditions

(1) $I=T_{\tau^{\prime}}(R)$ for some $\tau^{\prime} \in R$-prop;

(2) $R / I \in \mathscr{F}_{\tau}$.

Then $\mathscr{A}$ is nonempty since $T_{\tau}(R) \in \mathscr{A}$. Since $R$ is left noetherian, $\mathscr{A}$ has a maximal element $I_{0}$. Let $\tau_{0}=\chi\left(R / I_{0}\right)$. Then $\tau \leqq \tau_{0}$ since $R / I_{0} \in \mathscr{F}_{\tau}$. On the other hand, if $\tau_{0} \leqq \tau^{\prime} \in R$-prop then $I_{0} \leqq T_{\tau^{\prime}}(R)$ and 
$\mathscr{F}_{\tau^{\prime}} \subseteq \mathscr{F}_{\tau_{0}} \subseteq \mathscr{F}_{\tau}$ so that $R / T_{\tau^{\prime}}(R) \in \mathscr{F}_{\tau}$. But this implies that $T_{\tau^{\prime}}(R) \epsilon$ $\mathscr{A}$ and so $T_{\tau^{\prime}}(R) \subseteq I_{0}$, proving equality. Therefore $\tau_{0}=\tau^{\prime}$.

Lemma 2.3. If $U_{1}$ and $U_{2}$ are subsets of $R$-prop then

(1) $U_{1} \subseteq U_{2}$ implies that $d\left(U_{1}\right) \geqq d\left(U_{2}\right)$.

(2) $d\left(U_{1} \cup U_{2}\right)=d\left(U_{1}\right) \wedge d\left(U_{2}\right)$.

Proof. (1) follows directly from the definition. As for (2),

$$
\begin{aligned}
\mathscr{T}_{d\left(U_{1} \cup U_{2}\right)} & =\left\{M \mid \operatorname{wsupp}(M) \cap\left(U_{1} \cup U_{2}\right)=\varnothing\right\} \\
& =\left\{M \mid\left[\operatorname{wsupp}(M) \cap U_{1}\right] \cup\left[\operatorname{wsupp}(M) \cap U_{2}\right]=\varnothing\right\} \\
& =\left\{M \mid \operatorname{wsupp}(M) \cap U_{1}=\varnothing\right\} \cap\left\{M \mid \operatorname{wsupp}(M) \cap U_{2}=\varnothing\right\} \\
& =\mathscr{T}_{d\left(U_{1}\right)} \cap \mathscr{T}_{d\left(U_{2}\right)}=\mathscr{T}_{d\left(U_{1}\right) \wedge d\left(U_{2}\right)} .
\end{aligned}
$$

From Lemma 2.1(3) it is clear that the family $\{c(\tau) \mid \tau \in R$-tors $\}$ of subsets of $R$-prop is the base of a topology on $R$-prop, which we will call the order topology.

Proposition 2.4. For any $\tau \in R$-prop, $c(\tau)$ is quasicompact in the order topology. In particular, $R$-prop $=c(\xi)$ is quasicompact.

Proof. If $\left\{c\left(\tau_{i}\right) \mid i \in \Omega\right\}$ is an open cover of $c(\tau)$, then $\tau \in c\left(\tau_{k}\right)$ for some $k \in \Omega$ whence $c(\tau)=c\left(\tau_{k}\right)$ by Lemma 2.1(1).

Proposition 2.5. For any $\tau \in R$-prop, the closure of $\{\tau\}$ in the order topology on $R$-prop is $\left\{\tau^{\prime} \in R\right.$-prop $\left.\mid \tau^{\prime} \leqq \tau\right\}$.

Proof. By definition, $\tau^{\prime}$ belongs to the closure of $\{\tau\}$ if and only if every open neighborhood of $\tau^{\prime}$ intersects $\{\tau\}$. This clearly happens when $\tau^{\prime} \leqq \tau$. Conversely, if $\tau^{\prime} \not \leq$ then there exists an $M \in \mathscr{T}_{\tau^{\prime}} \mid \mathscr{T}_{\tau}$. Then $\tau^{\prime} \in c(\xi(M)), \tau \notin c(\xi(M))$, which shows that $c(\xi(M))$ is an open neighborhood of $\tau^{\prime}$ not containing $\tau$.

By Lemma 1.1, the family $\{c(\xi(R / I)) \mid I$ a left ideal of $R\}$ of subsets of $R$-prop also forms the base of a topology on $R$-prop. This topology is coarser than the order topology; we call it the finitary order topology on $R$-prop.

3. Prime torsion theories. The notion of a prime element of $R$-tors was first defined by Goldman [4] and has since been considered by several authors $[7,8,11]$. Of the equivalent definitions available in the literature, we will use the one from [7].

A left ideal $I$ of a ring $R$ is critical if and only if, for every left ideal $H$ of $R$ properly containing $I, R / H \in \mathscr{T}_{\chi(R / I)}$. It is easily shown 
that if $I$ is critical, it is meet-irreducible. Furthermore, if $R$ is commutative then $I$ is critical if and only if it is prime. We therefore define a torsion theory $\tau \in R$-tors to be prime if and only if $\tau=\chi(R / I)$ for some critical left ideal $I$ of $R$. The family of all prime elements of $R$-tors is called the left spectrum of $R$ and will be denoted by $R$-sp. If $\tau \in R$-sp then the family of all critical left ideals $I$ of $R$ with $\tau=\chi(R / I)$ will be denoted by $\operatorname{crit}(\tau)$.

EXAMPLE 3.1. Maximal left ideals of $R$ are trivially critical. Therefore, if $M$ is a simple left $R$-module, $\chi(M) \in R$-sp.

LemMA 3.2. Let $\tau \in R$-sp and $\tau_{1}, \tau_{2} \in R$-tors. Then

(1) $\tau=\tau_{1} \wedge \tau_{2}$ implies that $\tau=\tau_{1}$ or $\tau=\tau_{2}$.

(2) $\tau \geqq \tau_{1} \wedge \tau_{2}$ implies that $\tau \geqq \tau_{1}$ or $\tau \geqq \tau_{2}$.

Proof. (1) Assume that $\tau=\tau_{1} \wedge \tau_{2}$ where $\tau_{1}>\tau$ and $\tau_{2}>\tau$. If $I \in \operatorname{crit}(\tau)$ then $R / I$ belongs to neither $\mathscr{F}_{\tau_{1}}$ nor $\mathscr{F}_{\tau_{2}}$ and so we have nonzero modules $W_{j} / I=T_{\tau_{j}}(R / I), j=1,2$. On the other hand, $\left(W_{1} / I\right) \cap$ $\left(W_{2} / I\right)=T_{\tau}(R / I)=0$. This contradicts the fact that $I$ is meet irreducible.

(2) For $j=1$, 2, let $\tau_{j}^{\prime}=\tau \vee \tau_{j}$. If $\tau \geqq \tau_{1} \wedge \tau_{2}$ then $\tau=\tau_{1}^{\prime} \wedge \tau_{2}^{\prime}$ and so, by (1), $\tau=\tau_{j}^{\prime}$ for $j=1$ or $j=2$. This implies that $\tau \geqq \tau_{j}$ for that $j$.

For each ordinal $t$, define $[R \text {-sp }]_{t}$ by

(1) $[R \text {-sp }]_{0}=\{\tau \in R$-sp $\mid \tau$ is maximal $\}$.

(2) $[R \text {-sp }]_{t}=\left\{\tau \in R\right.$-sp $\mid \tau<\tau^{\prime} \in R$-sp $\left.\Rightarrow \tau^{\prime} \in \bigcup_{s<t}[R \text {-sp }]_{s}\right\}$. If there exists an ordinal $t$ with $[R \text {-sp }]_{t}=R$-sp then we say that $t$ is the Krull-Krause dimension of $R$-sp and that $R$-sp has Krull-Krause dimension. A proof analogous to that of [5, Proposition 1.2] then establishes

Proposition 3.3. The following conditions are equivalent for a $\operatorname{ring} R$ :

(1) $R$-sp has Krull-Krause dimension.

(2) $R$-sp satisfies the maximum condition.

Alternatively, for each ordinal $t$ define the torsion theory $\tau_{t}$ as follows:

(1) $\tau_{0}=\xi$.

(2) If $t$ is not a limit ordinal, $\tau_{t}=\xi\left(\left\{M \mid Q_{\tau_{t-1}}(M)\right.\right.$ is of finite length\}).

(3) If $t$ is a limit ordinal, $\tau_{t}=\mathrm{V}\left\{\tau_{s} \mid s<t\right\}$. If there exists an ordinal $t$ with $\chi=\tau_{t}$ then we say that $t$ is the Krull-Gabriel dimension 
of $R$-tors and that $R$-tors has Krull-Gabriel dimension. It is then easy to establish the following result [9, Corollaire 2.4]:

Proposition 3.4. If $R$-tors has Krull-Gabriel dimension then $R$-sp satisfies the minimum condition.

In particular we have

COROLLARY 3.5. If $R$ is left noetherian then $R$-sp satisfies the minimum condition.

For a left $R$-module $M$ we define the assassin ass (M) of $M$ to be the family of all $\tau \in R$-sp for which there exists an $m \in M$ with $(0: m) \in \operatorname{crit}(\tau)$.

Proposition 3.6.

(1) If $M=\mathrm{U} M_{i}$ then ass $(M)=\mathrm{U}$ ass $\left(M_{i}\right)$.

(2) If $I \in \operatorname{crit}(\tau)$ then for all $0 \neq{ }_{R} N \subseteq R / I$, ass $(N)=\{\tau\}$.

(3) If $N \cong M$ then ass $(N) \subseteq$ ass $(M) \cong \operatorname{ass}(N) \cup \operatorname{ass}(M / N)$.

(4) If $M=\bigoplus M_{i}$ then ass $(M)=\mathrm{U}$ ass $\left(M_{i}\right)$.

(5) If $N$ is a large submodule of $M$ then ass $(N)=\operatorname{ass}(M)$.

Proof. Parts (1)-(4) follow from [11, Proposition 3.1]. As for part (5), ass $(N) \leqq$ ass (M) by (3). Conversely, assume that $\tau \in \operatorname{ass}(M)$. Then there exists an $r \in R$ with $0 \neq r m \in N$, where $(0: m) \in \operatorname{crit}(\tau)$. Furthermore, $(0: r m)=((0: m): r)$. Since $(0: m) \in \operatorname{crit}(\tau)$, we have $(0: \mathrm{rm}) \in \operatorname{crit}(\tau)$ by [6, Proposition 2.8] and so $\tau \in \operatorname{ass}(N)$.

Proposition 3.7. The following conditions are equivalent:

(1) $M \neq 0$ implies that ass $(M) \neq \varnothing$.

(2) If $I$ is a proper left ideal of $R$ then there exists an $r \in R$ with $(I: r)$ critical.

Proof. $(1) \Rightarrow(2)$ : Let $I$ be a proper left ideal of $R$. Then by (1) there exists a $\tau \in$ ass $(R / I)$ and so there exists an $r \in R$ with $(I: r)=$ $(0: r+I) \in \operatorname{crit}(\tau)$.

$(2) \Rightarrow(1):$ Let $M \neq 0$ and pick $0 \neq m \in M$. Then by (2) there exists an $r \in R$ with $(0: r m)=((0: m): r)$ critical. If $\tau=\chi(R m)$ then $\tau \in \operatorname{ass}(M)$.

This condition is satisfied if $R$ is left noetherian. In fact, we have the slightly stronger result.

Ppoposition 3.8. If $M$ is a nonzero noetherian module then 
ass $(M)$ is a nonempty finite set.

Proof. By [11, Proposition 3.3] and [4, Theorem 6.14].

COROLlary 3.9. If $R$ is left noetherian then $R$-sp is a dense subset of $R$-prop in the order topology.

Proof. Let $\tau \in R$-prop and let $M$ be an injective cogenerator of $\mathscr{F}_{\tau}$. If $0 \neq m \in M$ then $R m$ is noetherian. Let $\tau^{\prime} \in \operatorname{ass}(R m) \leqq \operatorname{ass}(M)$. Then $\mathscr{F}_{\tau^{\prime}} \subseteq \mathscr{F}_{\text {: }}$ and so $\tau^{\prime} \in c(\tau) \cap R$-sp.

Proposition 3.10. If $R$ is left noetherian then the homomorphism $\psi: M \rightarrow \Pi\left\{Q_{\tau}(M) \mid \tau \in \operatorname{ass}(M)\right\}$ defined by $m \mapsto\left\langle m \hat{\tau}_{M}\right\rangle$ is a monomorphism.

Proof. If $0 \neq K=\operatorname{ker}(\psi)$ then by Propositions 3.8 and 3.6(3) there exists a $\tau \in \operatorname{ass}(K) \subseteq$ ass $(M)$. If $k \in K$ with $(0: k) \in \operatorname{crit}(\tau)$ then $R k \in \mathscr{F}_{\tau}$ so $k \hat{\tau}_{M} \neq 0$, contradicting the fact that $K=\bigcap\left\{\operatorname{ker}\left(\hat{\tau}_{M}\right) \mid \tau \epsilon\right.$ $\operatorname{ass}(M)\}$.

For a module $M$ we define the support of $M$ by $\operatorname{supp}(M)=$ wsupp $(M) \cap R$-sp. We then have the following result analogous to Lemma 1.2, again directly from the definition.

Lemma 3.11. For a module $M$,

(1) $M=\Sigma M_{i}$ implies that $\operatorname{supp}(M)=\bigcup \operatorname{supp}\left(M_{i}\right)$.

(2) $N \subseteq M$ implies that $\operatorname{supp}(M)=\operatorname{supp}(N) \cup \operatorname{supp}(M / N)$.

It is clear that, for any module $M$, ass $(M) \subseteq \operatorname{supp}(M)$. Therefore, as a corollary to Proposition 3.8 we have

Proposition 3.12. If $R$ is left noetherian then $M=0$ if and only if $\operatorname{supp}(M)=\varnothing$.

4. The order topologies induced on $R$-sp. The [finitary] order topology defined in $\$ 2$ induces a topology on $R$-sp, a basis for which is the family of sets $c^{\prime}(\tau)=c(\tau) \cap R$-sp, for each $\tau \in R$-tors [resp. $\tau=$ $\xi(R / I)]$.

Lemma 4.1. If $\tau_{1}, \tau_{2} \in R$-sp then $c^{\prime}\left(\tau_{1} \wedge \tau_{2}\right)=c^{\prime}\left(\tau_{1}\right) \cup c^{\prime}\left(\tau_{2}\right)$.

Proof. That $c^{\prime}\left(\tau_{1}\right) \cup c^{\prime}\left(\tau_{2}\right) \subseteq c^{\prime}\left(\tau_{1} \wedge \tau_{2}\right)$ follows from Lemma 2.1(2). 
Conversely, if $\tau \in c^{\prime}\left(\tau_{1} \wedge \tau_{2}\right)$ then $\tau \in c^{\prime}\left(\tau_{1}\right) \cup c^{\prime}\left(\tau_{2}\right)$ by Lemma 3.2(2).

Lemma 4.2. If $\tau \in R$-tors and $M \in \mathscr{F}_{\tau}$ then ass $(M) \subseteq c^{\prime}(\tau)$.

Proof. If $\tau^{\prime} \in \operatorname{ass}(M)$ then there exists an $0 \neq m \in M$ with $\tau^{\prime}=$ $\chi(R m)$. Since $M \in \mathscr{F}_{\tau}, R m \in \mathscr{F}_{\tau}$ and so $\tau \leqq \tau^{\prime}$.

If $U \subseteq R$-sp, then in general $d(U) \notin R$-sp.

Proposition 4.3. Let $\tau \in R$-tors satisfy

$\left(^{*}\right)$ Every $0 \neq M \in \mathscr{F}_{\tau}$ has a nonzero noetherian submodule. Then $d c^{\prime}(\tau)=\tau$.

Proof. Clearly $d c^{\prime}(\tau) \geqq \tau$. Conversely assume that $M \in \mathscr{T}_{d c^{\prime}(\tau)} \backslash \mathscr{T}_{\tau}$. Then $0 \neq M / T_{\tau}(M)$ and so there exists a $\tau^{\prime} \in \operatorname{ass}\left(M / T_{\tau}(M)\right)$ by $\left({ }^{*}\right)$ and Proposition 3.8. Furthermore, $M \notin \mathscr{T}_{\tau^{\prime}}$ since otherwise we would have $M / T_{\tau}(M) \in \mathscr{T}_{\tau^{\prime}}$ which contradicts the definition of $\tau^{\prime}$. Therefore $\tau^{\prime} \in$ supp $(M)$. On the other hand, $\mathscr{F}_{\tau^{\prime}} \subseteq \mathscr{F}_{\tau}$ by construction and so $\tau^{\prime} \epsilon$ $c^{\prime}(\tau)$ whence $\tau^{\prime} \notin \operatorname{supp}(M)$ by the choice of $M$. From this contradiction we deduce that $d c^{\prime}(\tau) \leqq \tau$ and so we have equality.

We have thus seen that, particularly for the case of a left noetherian ring $R$, the order topology on $R$-sp exhibits various "nice" features of the Zariski topology on the spectrum $\operatorname{spec}(R)$ of a commutative ring $R$. It is the finitary order topology, however, which reduces to the Zariski topology in the case that $R$ is commutative.

Proposition 4.4. If $R$ is commutative then $R$-sp with the finitary order topology is homeomorphic to spec $(R)$ with the Zariski topology.

Proof. Define the function $h: \operatorname{spec}(R) \rightarrow R$-sp by $P \mapsto \chi(R / P)$. Since the critical left ideals of a commutative ring $R$ are precisely the prime ideals of $R$ [7] the function $h$ is clearly a surjection. Furthermore, by [4, Proposition 5.2],

$$
T_{\chi(R \mid P)}(M)=\left\{m \in M \mid r^{n} m=0 \text { for some } r \in R \backslash P \text { and some integer } n\right\}
$$

which shows that $h$ is a bijection.

If $I$ is an ideal of $R$ and $V(I)=\{P \in \operatorname{spec}(R) \mid I \subseteq P\}$ is a subset of spec $(R)$ closed in the Zariski topology, then $h(V(I))=\{\chi(R / P) \in$ $R$-sp $\mid \chi(R / P) \leqq \chi(R / I)\}$ which is closed in the finitary order topology on $R$-sp by Proposition 2.5. Conversely, inverse images under $h$ of subsets of $R$-sp closed in the finitary order topology are clearly closed in $\operatorname{spec}(R)$. Therefore, $h$ is a homeomorphism. 


\section{The reverse order topology on $R$-sp.}

Proposition 5.1. If $R$ is left noetherian then c'd is a closure operator on $R$-sp.

Proof. Clearly $c^{\prime} d(\varnothing)=\varnothing$. By definition, $c^{\prime} d(U) \supseteqq U$ for every subset $U$ of $R$-sp. In particular, if $U \subseteq R$-sp then $c^{\prime} d(U) \leqq c^{\prime} d c^{\prime} d(U)$. Conversely, if $\tau \in c^{\prime} d c^{\prime} d(U)$ then $d c^{\prime} d(U) \leqq \tau$ and so by Proposition 4.3, $d(U) \leqq \tau$ which implies that $\tau \in c^{\prime} d(U)$. Therefore $c^{\prime} d c^{\prime} d(U)=c^{\prime} d(U)$. Finally, by Lemmas 2.3 and 4.1, $c^{\prime} d\left(U_{1} \cup U_{2}\right)=c^{\prime}\left(d\left(U_{1}\right) \wedge d\left(U_{2}\right)\right)=$ $c^{\prime} d\left(U_{1}\right) \cup c^{\prime} d\left(U_{2}\right)$.

Proposition 5.1 shows that, for a left noetherian ring $R$, we have another topology on $R$-sp which is opposite to the order topology in the sense that the open sets are precisely the sets of the form $R$-sp $\mid c^{\prime}(\tau)$ for some $\tau \in R$-tors. We call this topology the reverse order topology on $R$-tors.

LEMMA 5.2. Let $R$ be a left noetherian ring. Then for any module $M$, supp $(M)$ is open in the reverse order topology on $R$-sp.

Proof. Let $\tau=\Lambda\left\{\tau^{\prime} \mid M \in \mathscr{T}_{\tau^{\prime}}\right\}$. Then $M \in \mathscr{T}_{\tau}$ so $\tau \notin \operatorname{supp}(M)$. If $\tau^{\prime \prime} \in R$-sp $\mid c^{\prime}(\tau)$ then $M \notin \mathscr{T}_{\tau^{\prime \prime}}$ so $\tau^{\prime \prime} \in \operatorname{supp}(M)$. The converse is trivial. Hence $\operatorname{supp}(M)=R$-sp $\mid c^{\prime}(\tau)$ is open.

We now develop another method for characterizing the reverse order topology on $R$-sp. To this end define a function

$$
\text { subsets of } R \text {-sp } \stackrel{e}{\longrightarrow} R \text {-tors }
$$

by $e: U \mapsto \chi(\{N \mid$ ass $(N) \subseteq U\})$. Then

Lemma 5.3. If $U_{1}$ and $U_{2}$ are subsets of $R$-sp then

(1) $U_{1} \subseteq U_{2}$ implies that $e\left(U_{1}\right) \geqq e\left(U_{2}\right)$.

(2) $e\left(U_{1} \cup U_{2}\right)=e\left(U_{1}\right) \wedge e\left(U_{2}\right)$.

Proof. (1) follows directly from the definition. As for (2),

$$
\begin{aligned}
e\left(U_{1} \cup U_{2}\right) & =\chi\left(\left\{N \mid \operatorname{ass}(N) \subseteq U_{1} \cup U_{2}\right\}\right) \\
& =\chi\left(\left\{N \mid \operatorname{ass}(N) \subseteq U_{1}\right\}\right) \wedge \chi\left(\left\{N \mid \operatorname{ass}(N) \subseteq U_{2}\right\}\right) \\
& =e\left(U_{1}\right) \wedge e\left(U_{2}\right) .
\end{aligned}
$$

Lemma 5.4. If $U$ is a subset of $R$-sp then $d(U) \geqq e(U)$.

Proof. Let $M \in \mathscr{T}_{e(U)}$ and let $\tau \in U$. Then for any $I \in \operatorname{crit}(\tau)$, ass $(E(R / I))=\{\tau\}$ by Proposition 3.6. Therefore, $\operatorname{Hom}_{R}(M, E(R / I))=0$ 
and so $M \in \mathscr{T}_{\tau}$. Hence $M \in \mathscr{T}_{e(U)}$ implies that $M \in \bigcap\left\{\mathscr{T}_{:} \mid \tau \in U\right\}=$ $\mathscr{T}_{d(U)}$.

We now prove a result analogous to Proposition 4.3.

Proposition 5.5. Let $\tau \in R$-tors satisfy

(**) Every $0 \neq M \in \mathscr{T}_{\tau}$ has a nonzero noetherian submodule. Then $e c^{\prime}(\tau)=\tau$.

Proof. To prove that $e c^{\prime}(\tau)=\tau$ it suffices to show that $M \in \mathscr{F}_{\tau}$ if and only if ass $(M) \cong c^{\prime}(\tau)$. Assume that ass $(M) \subseteq c^{\prime}(\tau)$ and that $M \notin \mathscr{F}_{\tau}$. Then $T_{\tau}(M) \neq 0$ and so by $\left({ }^{* *}\right)$ and Proposition 3.8 there exists a $\tau^{\prime} \in \operatorname{ass}\left(T_{\tau}(M)\right) \subseteq$ ass $(M)$. Therefore, $\tau^{\prime} \in c^{\prime}(\tau)$ by assumption. But $T_{\tau}(M) \in \mathscr{T}_{\tau}$ implies that ass $\left(T_{\tau}(M)\right) \cap c^{\prime}(\tau)=\varnothing$ since $\tau \leqq d c^{\prime}(\tau)$ and ass $(M) \leqq \operatorname{supp}(M)$. This yields a contradiction which shows that we must have $M \in \mathscr{F}_{\tau}$. The reverse implication follows directly from Lemma 4.2.

Proposition 5.6. If $R$ is left noetherian then

(1) $d c^{\prime}=e c^{\prime}=$ identity on $R$-tors.

(2) $\quad c^{\prime} d=c^{\prime} e$.

Proof. (1) follows directly from Propositions 5.5 and 4.3. As for (2), by definition we have $c^{\prime} e(U) \supseteqq U$ for any subset $U$ of $R$-sp and so, in particular, for any such $U$ we have $c^{\prime} e(U) \subseteq c^{\prime} e c^{\prime} e(U)$. Conversely if $\tau \in c^{\prime} e c^{\prime} e(U)$ then $e c^{\prime} e(U) \leqq \tau$. By part (1), $e c^{\prime} e(U)=e(U)$ and so $e(U) \leqq \tau$ which implies that $\tau \in c^{\prime} e(U)$. This proves that $c^{\prime} e(U)=$ $c^{\prime} e c^{\prime} e(U)$.

Now let $U \subseteq R$-sp. Then $c^{\prime} d(U)=c^{\prime} d c^{\prime} d(U)=c^{\prime} e c^{\prime} d(U)$ by part (1). Furthermore, $c^{\prime} e c^{\prime} d(U) \supseteqq c^{\prime} e(U)$ since $c^{\prime} d(U) \supseteqq U$. On the other hand, $c^{\prime} e(U)=c^{\prime} e c^{\prime} e(U)=c^{\prime} d c^{\prime} e(U) \supseteqq c^{\prime} d(U)$ by a similar argument and so we have $c^{\prime} e(U)=c^{\prime} d(U)$.

Thus we see that the reverse order topology also resembles the Zariski topology although it "goes the other way". In particular, the construction of the reverse order topology is formally the same as the classical "hull-kernel" construction of the Zariski topology.

6. The Gillman topology on $R$-sp. If $R$ is left noetherian we can define another function

$$
\text { subsets of } R \text {-sp } \stackrel{g}{\longrightarrow} R \text {-tors }
$$

by $g: U \mapsto \bigvee\left\{d\left(U^{\prime}\right) \mid U \subseteq U^{\prime} \subseteq R\right.$-sp and $U^{\prime}$ is open in the reverse order topology on $R$-sp\}. 
Lemma 6.1. If $R$ is left noetherian then for $U_{1}, U_{2} \subseteq R$-sp,

(1) $U_{1} \subseteq U_{2}$ implies that $g\left(U_{1}\right) \geqq g\left(U_{2}\right)$.

(2) $U_{1} \subseteq c^{\prime} g\left(U_{1}\right)$.

Proof. (1) follows directly from the definition. As for (2), if $U^{\prime}$ is a neighborhood of $U_{1}$ in the reverse order topology on $R$-sp then $d\left(U^{\prime}\right)=\Lambda U^{\prime}$ is the largest torsion theory less than or equal to every element of $U^{\prime}$. In particular, if $\tau \in U$ then $\tau \geqq d\left(U^{\prime}\right)$. Since $\vee d\left(U^{\prime}\right)$ is the smallest torsion theory greater than or equal to all of the $d\left(U^{\prime}\right)$, $\tau \geqq \vee d\left(U^{\prime}\right)=g\left(U_{1}\right)$. Thus $\tau \in c^{\prime} g\left(U_{1}\right)$ for all $\tau \in U_{1}$, proving (2).

Lemma 6.2. Let $R$ be left noetherian and let $\tau \in R$-sp. Then for any $U \subseteq R$-sp, $U \cap c^{\prime}(\tau) \neq \varnothing$ implies $\tau \in c^{\prime} g(U)$.

Proof. To show that $\tau \in c^{\prime} g(U)$ we have to show that for every open neighborhood $U^{\prime}$ of $U, d\left(U^{\prime}\right) \leqq \tau$. Let $U^{\prime}$ be such an open neighborhood and let $V^{\prime}=R$-sp $\backslash U^{\prime}$. Then $d\left(V^{\prime}\right) \wedge d\left(U^{\prime}\right) \leqq \tau$ so by Lemma 3.2 either $d\left(V^{\prime}\right) \leqq \tau$ or $d\left(U^{\prime}\right) \leqq \tau$. But $d\left(V^{\prime}\right) \leqq \tau$ implies that $\tau \in$ $c^{\prime} d\left(V^{\prime}\right)=V^{\prime}$ (since $V^{\prime}$ is closed in the reverse orde topology) whence $c(\tau) \cong V^{\prime}$, contradicting the hypothesis that $U \cap c(\tau) \neq \varnothing$. Therefore, we must have $d\left(U^{\prime}\right) \leqq \tau$.

Proposition 6.3. If $R$ is left noetherian then $c^{\prime} g$ is a closure operator on $R$-sp.

Proof. For any subset $U$ of $R$-sp, $U \subseteq c^{\prime} g(U)$ by Lemma 6.1. Furthermore, it is clear that $c^{\prime} g(\varnothing)=\varnothing$. Also, by Lemma 6.1, $c^{\prime} g(U) \leqq c^{\prime} g c^{\prime} g(U)$. Conversely, $c^{\prime} g c^{\prime} g(U) \cap U \neq \varnothing$ and so, by Lemma 6.2, $g c^{\prime} g(U) \in c^{\prime} g(U)$, i.e., $g c^{\prime} g(U) \geqq g(U)$. By Lemma 2.1 this implies that $c^{\prime} g(U) \supseteqq c^{\prime} g c^{\prime} g(U)$ and so we have equality.

Finally we have to show that $c^{\prime} g\left(U_{1} \cup U_{2}\right)=c^{\prime} g\left(U_{1}\right) \cup c^{\prime} g\left(U_{2}\right)$. For $i=1,2, U_{i} \subseteq U_{1} \cup U_{2}$ and so $g\left(U_{i}\right) \geqq g\left(U_{1} \cup U_{2}\right)$. Thus $g\left(U_{1}\right) \wedge g\left(U_{2}\right) \geqq$ $g\left(U_{1} \cup U_{2}\right)$. By Lemma 2.1 this implies that $c^{\prime} g\left(U_{1}\right) \cup c^{\prime} g\left(U_{2}\right)=c^{\prime}\left(g\left(U_{1}\right) \wedge\right.$ $\left.g\left(U_{2}\right)\right) \cong c^{\prime} g\left(U_{1} \cup U_{2}\right)$. Conversely suppose that $\tau \in c^{\prime} g\left(U_{1} \cup U_{2}\right)$. To show that $\tau \in c^{\prime}\left(g\left(U_{1}\right) \wedge g\left(U_{2}\right)\right)$ it suffices to show that $\tau \geqq g\left(U_{1}\right)$ or $\tau \geqq g\left(U_{2}\right)$. Assume neither holds. Then $\tau \notin c^{\prime} g\left(U_{1}\right) \cap c^{\prime} g\left(U_{2}\right)$. In particular, there then exist open neighborhoods $U_{i}^{\prime}$ of $U_{i}(i=1,2)$ with $\tau \notin c^{\prime} d\left(U_{i}^{\prime}\right)$. Therefore, $\tau \notin c^{\prime} d\left(U_{1}^{\prime}\right) \cup c^{\prime} d\left(U_{2}^{\prime}\right)=c^{\prime} d\left(U_{1}^{\prime} \cup U_{2}^{\prime}\right)$. But $U_{1}^{\prime} \cup U_{2}^{\prime}$ is an open neighborhood of $U_{1} \cup U_{2}$ and so $c^{\prime} d\left(U_{1}^{\prime} \cup U_{2}^{\prime}\right)$ contains $\tau$ by hypothesis. From this contradiction we have $\tau \in c^{\prime}\left(g\left(U_{1}\right) \wedge g\left(U_{2}\right)\right)=$ $c^{\prime} g\left(U_{1}\right) \cup c^{\prime} g\left(U_{2}\right)$.

If $R$ is left noetherian, the closure operator $c^{\prime} g$ thus defines a topology on $R$-sp, which we will call the Gillman topology since the 
above construction is based on the construction in [3]. We now use the Gillman topology to characterize the reverse order topology.

Proposition 6.4. The following conditions are equivalent for a left noetherian ring $R$ :

(1) $R$-sp is a $T_{2}$-space under the reverse order topology.

(2) $R$-sp is a $T_{1}$-space under the Gillman topology.

Proof. $\quad(1) \Longrightarrow(2)$. To show that $R$-sp is a $T_{1}$-space under the Gillman topology we have to show that for every $\tau \in R$-sp, $\{\tau\}=$ $c^{\prime} g(\{\tau\})$. By Lemma 6.1 we know that $\{\tau\} \subseteq c^{\prime} g(\{\tau\})$. Conversely, assume that $\tau \neq \tau^{\prime} \in c^{\prime} g(\{\tau\})$. Then $\tau^{\prime} \geqq g(\{\tau\})$ and so $\tau^{\prime} \geqq d\left(U^{\prime}\right)$ for any open neighborhood $U^{\prime}$ of $\tau$. This means that $\tau^{\prime} \in c^{\prime} d\left(U^{\prime}\right)$ for any open neighborhood $U^{\prime}$ of $\tau$. Since $c^{\prime} d\left(U^{\prime}\right)$ is the closure of $U^{\prime}$ in the reverse order topology, this means that there is no neighborhood of $\tau^{\prime}$ which does not intersect a neighborhood of $\tau$, contradicting the fact that $R$-sp is a $T_{2}$-space under the reverse order topology.

$(2) \Rightarrow(1)$ : Let $\tau \neq \tau^{\prime} \in R$-sp. Then $\tau^{\prime} \notin c^{\prime} g(\{\tau\})$ by (2) and so there exists some proper open neighborhood $U^{\prime}$ of $\tau$ with $\tau^{\prime} \notin c^{\prime} d\left(U^{\prime}\right)$. Then $R \mid c^{\prime} d\left(U^{\prime}\right)$ is an open neighborhoood of $\tau^{\prime}$ not intersecting $U^{\prime}$. This proves that $R$-sp is a $T_{2}$-space under the reverse order topology.

\section{REFERENCES}

1. J. Beachy, On maximal torsion theories, Canad. J. Math., 25 (1973), 712-726.

2. S. E. Dickson, A torsion theory for abelian categories, Trans. Amer. Math. Soc., 121 (1966), 223-235.

3. L. Gillman, Rings with Hausdorff structure space, Fund. Math., 45 (1957), 1-16.

4. O. Goldman, Rings and modules of quotients, J. Algebra, 13 (1969), 10-47.

5. G. Krause, On fully left bounded left noetherian rings, J. Algebra, 23 (1972), 88-99.

6. J. Lambek, Torsion Theories, Additive Semantics, and Rings of Quotients, Lecture notes in Mathematics \#177, Berlin: Springer-Verlag, 1971.

7. J. Lambek and G. Michler, The torsion theory at a prime ideal of a right noetherian ring, J. Algebra, 25 (1973), 364-389.

8. N. Popescu, Le spectre à gauche d'un anneau, J. Algebra, 18 (1971), 213-228.

9. J. Raynaud, Localisations stables à droite et anneaux semi-noetheriens à droite, C. R. Acad. Sc. Paris, 275 (1972), A13-A16.

10. B. Stenström, Rings and Modules of Quotients, Lecture notes in Mathematics \#237, Berlin: Springer-Verlag, 1972.

11. H. Storrer, On Goldman's Primary Decomposition, Lectures on rings and modules, Lecture notes in Mathematics \#246, Berlin: Springer-Verlag, 1972.

Received December 12, 1972. This research was partially supported by the National Research Council of Canada.

MeGILl UNIVERSITY

Current address: University of Haifa

Haifa, Israel 


\section{PACIFIC JOURNAL OF MATHEMATICS}

\section{EDITORS}

RICHARD ARENS (Managing Editor)

University of California

Los Angeles, California 90024
J. DUGUNDJI*

Department of Mathematics

University of Southern California

Los Angeles, California 90007

D. Gilbarg and J. Milgram

Stanford University

Stanford, California 94305
University of Washington

Seattle, Washington 98105

ASSOCIATE EDITORS
E. F. BECKENBACH
B. H. NeumanN
F. WoLF
K. YosHIDA

\section{SUPPORTING INSTITUTIONS}

\author{
UNIVERSITY OF BRITISH COLUMBIA \\ CALIFORNIA INSTITUTE OF TECHNOLOGY \\ UNIVERSITY OF CALIFORNIA \\ MONTANA STATE UNIVERSITY \\ UNIVERSITY OF NEVADA \\ NEW MEXICO STATE UNIVERSITY \\ OREGON STATE UNIVERSITY \\ UNIVERSITY OF OREGON \\ OSAKA UNIVERSITY
}

\author{
UNIVERSITY OF SOUTHERN CALIFORNIA \\ STANFORD UNIVERSITY \\ UNIVERSITY OF TOKYO \\ UNIVERSITY OF UTAH \\ WASHINGTON STATE UNIVERSITY \\ UNIVERSITY OF WASHINGTON
* * * *
AMERICAN MATHEMATICAL SOCIETY \\ NAVAL WEAPONS CENTER
}

The Supporting Institutions listed above contribute to the cost of publication of this Journal, but they are not owners or publishers and have no responsibility for its content or policies.

Mathematical papers intended for publication in the Pacific Journal of Mathematics should be in typed form or offset-reproduced, (not dittoed), double spaced with large margins. Underline Greek letters in red, German in green, and script in blue. The first paragraph or two must be capable of being used separately as a synopsis of the entire paper. Items of the bibliography should not be cited there unless absolutely necessary, in which case they must be identified by author and Journal, rather than by item number. Manuscripts, in duplicate if possible, may be sent to any one of the four editors. Please classify according to the scheme of Math. Rev. Index to Vol. 39. All other communications to the editors should be addressed to the managing editor, or Elaine Barth, University of California, Los Angeles, California, 90024.

100 reprints are provided free for each article, only if page charges have been substantially paid. Additional copies may be obtained at cost in multiples of 50 .

The Pacific of Journal Mathematics is issued monthly as of January 1966. Regular subscription rate: $\$ 72.00$ a year (6 Vols., 12 issues). Special rate: $\$ 36.00$ a year to individual members of supporting institutions.

Subscriptions, orders for back numbers, and changes of address should be sent to Pacific Journal of Mathematics, 103 Highland Boulevard, Berkeley, California, 94708.

PUBLISHED BY PACIFIC JOURNAL OF MATHEMATICS, A NON-PROFIT CORPORATION

Printed at Kokusai Bunken Insatsusha (International Academic Printing Co., Ltd.), 270, 3-chome Totsuka-cho, Shinjuku-ku, Tokyo 160, Japan.

* C. R. DePrima California Institute of Technology, Pasadena, CA 91109, will replace J. Dugundji until August 1974.

Copyright (C) 1973 by Pacific Journal of Mathematics

Manufactured and first issued in Japan 


\section{Pacific Journal of Mathematics}

\section{Vol. 51, No. $2 \quad$ December, 1974}

Robert F. V. Anderson, Laplace transform methods in multivariate spectral theory .................................................. 339

William George Bade, Two properties of the Sorgenfrey plane . . . . . . . . . . . . 349

John Robert Baxter and Rafael Van Severen Chacon, Functionals on continuous

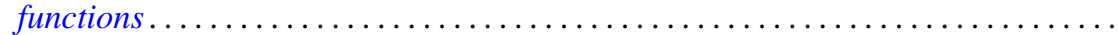

Phillip Wayne Bean, Helly and Radon-type theorems in interval convexity

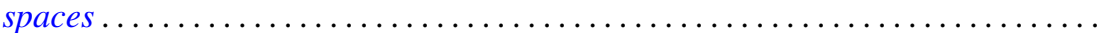

James Robert Boone, On k-quotient mappings $\ldots \ldots \ldots \ldots \ldots \ldots \ldots \ldots \ldots$

Ronald P. Brown, Extended prime spots and quadratic forms . . . . . . . . . . . .

William Hugh Cornish, Crawley's completion of a conditionally upper continuous lattice .............................................

Robert S. Cunningham, On finite left localizations ...................

Robert Jay Daverman, Approximating polyhedra in codimension one spheres

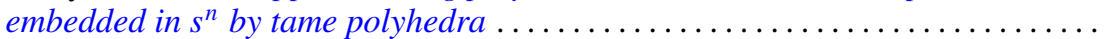

Burton I. Fein, Minimal splitting fields for group representations . . . . . . . . . . . .

Peter Fletcher and Robert Allen McCoy, Conditions under which a connected

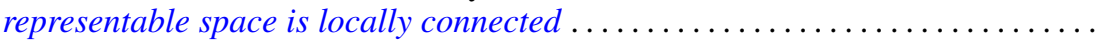

Jonathan Samuel Golan, Topologies on the torsion-theoretic spectrum of a noncommutative ring...

Manfred Gordon and Edward Martin Wilkinson, Determinants of Petrie matrices.

Alfred Peter Hallstrom, A counterexample to a conjecture on an integral condition for determining peak points (counterexample concerning peak points)........

E. R. Heal and Michael Windham, Finitely generated $F$-algebras with applications to Stein manifolds.

Denton Elwood Hewgill, On the eigenvalues of a second order elliptic operator in an unbounded domain ............................

Charles Royal Johnson, The Hadamard product of $A$ and $A^{*}$.

Darrell Conley Kent and Gary Douglas Richardson, Regular completions of Cauchy spaces.

Alan Greenwell Law and Ann L. McKerracher, Sharpened polynomial approximation

Bruce Stephen Lund, Subalgebras of finite codimension in the algebra of analytic functions on a Riemann surface. .

Robert Wilmer Miller, TTF classes and quasi-generators . .

Roberta Mura and Akbar H. Rhemtulla, Solvable groups in which every maximal partial order is isolated ....

Isaac Namioka, Separate continuity and joint continuity...

Alan Saleski, Entropy of self-homeomorphisms of statistical pseudo-metric

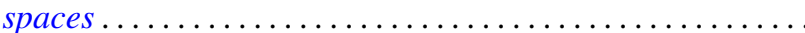

H. A. Seid, Cyclic multiplication operators on $L_{p}$-spaces .....

H. B. Skerry, On matrix maps of entire sequences ............

John Brendan Sullivan, A proof of the finite generation of invariants of a normal

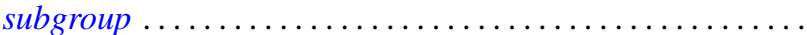

John Griggs Thompson, Nonsolvable finite groups all of whose local subgroups are

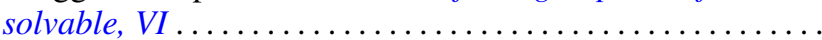

